\title{
Vigabatrin in the treatment of epilepsy: a long-term follow-up study
}

\author{
A TARTARA, R MANNI, C A GAlimberti, J P MUMFORD, A IUdice, \\ E PERUCCA
}

From the Institute of Neurology "C. Mondino", University of Pavia, the Merrell-Dow Research Institute, Strasbourg, France and the Department of Medical Pharmacology, University of Pavia, Pavia, Italy

SUMMARY Twenty-five adult epileptic patients who had shown a satisfactory clinical response to add-on vigabatrin under placebo-controlled conditions continued on long-term treatment for up to 47 months (median 22 months). The initially favourable therapeutic response was generally maintained throughout the observation period with an overall good clinical tolerance. No evidence of neurotoxicity was detected by multimodal evoked potentials monitoring.

In recent years, the hypothesis that enhancement of GABA-ergic transmission may have important therapeutic effects in epilepsy has received strong experimental support by the demonstration that the GABA-transaminase inhibitor vigabatrin (VGB), which raises brain GABA levels in animals and in man, ${ }^{1}$ is a very effective anticonvulsant in the clinical setting. Short-term placebo-controlled studies have shown consistently that the drug induces a greater than $50 \%$ decrease in fit frequency in approximately $60 \%$ of patients with otherwise "intractable" seizure disorders. $^{2-6}$ In order to assess fully the potential usefulness of VGB in the management of epilepsy, however, its anticonvulsant efficacy and clinical tolerability also need to be evaluated during chronic treatment. In the only long-term study published to date, the investigators from the U.S. Collaborative Multicenter Study ${ }^{7}$ have recently reported on 66 patients with complex partial seizures maintained on VGB for 4 to 25 months (median 16.7 months); among the 41 patients who had shown a good initial response to the drug, most were found to retain this effect throughout the full treatment period, suggesting that tolerance to the antiepileptic action does not develop. In the present paper, we describe the results of longterm treatment with VGB in 25 epileptic patients whose responsiveness to the drug had been demon-

Presented in part at the 40th Annual Meeting of the American Academy of Neurology, Cincinnati, Ohio, April 1988.

Address for reprint requests: Prof Tartara, Neurology Clinic, University of Pavia, Via Palestro, 27100 Pavia, Italy.

Received 21 June 1988 and in revised form 4 October 1988. Accepted 9 October 1988 strated previously under placebo-controlled conditions. $^{58}$

\section{Methods}

Patient selection The study was conducted in 25 epileptic patients attending the Epilepsy Clinic of the Department of Neurology of the University of Pavia, who fulfilled the following inclusion criteria: age between 18 and 65 years; epilepsy uncontrolled by conventional treatment, irrespectively of seizure type; treatment with no more than two conventional antiepileptic drugs; no evidence of hepatic, renal, cardiac or progressive neurological disease; no major psychiatric disorder; previous satisfactory clinical response to VGB, defined as an at least $50 \%$ reduction in seizure frequency or as a clinically important improvement in quality of life due to decreased seizure duration or severity. All the eligible patients fulfilling these criteria gave their consent to enter the study and were enrolled.

In 13 patients, previous response to VGB was established on the basis of the results of a double-blind randomised trial during which add-on VGB (2-3 g daily) and placebo were given for 7-week periods to a total of 20 patients. ${ }^{5}$ The remaining 12 patients were included after a 9-month singleblind study in 13 patients, which consisted of a 3-month baseline (1-month observation followed by 2 months placebo) and a subsequent 6-month period during which vigabatrin dosage ( $2-4 \mathrm{~g}$ daily) was adjusted according to clinical response.

Details of the patients included in the study are given in table 1 .

Study design Each patient was maintained on long-term treatment with VGB at a dosage up to $4 \mathrm{~g}$ daily (four $500 \mathrm{mg}$ tablets b.i.d.) adjusted according to the clinical response. In nine patients, a VGB-free period of 11 days to 42 weeks (median 11 weeks) elapsed between the placebo-controlled evaluation and the start of long-term treatment. Twenty-four 
Table 1 Age, sex, seizure type

\begin{tabular}{|c|c|c|c|c|c|c|c|c|}
\hline \multirow[b]{2}{*}{$\begin{array}{l}\text { Patient } \\
\text { Code }\end{array}$} & \multirow[b]{2}{*}{ Sex } & \multirow[b]{2}{*}{$\begin{array}{l}\text { Age } \\
\text { (years) }\end{array}$} & \multirow[b]{2}{*}{$\begin{array}{l}\text { Seizure } \\
\text { type }\end{array}$} & \multirow[b]{2}{*}{$\begin{array}{l}\text { Associated drugs } \\
\text { (mg/day) }\end{array}$} & \multirow{2}{*}{$\begin{array}{l}\text { Duration of } \\
\text { VGB treatment } \\
\text { (months) }\end{array}$} & \multirow{2}{*}{$\begin{array}{l}\text { Daily VGB } \\
\text { dosage during } \\
\text { last } 2 \text { months } \\
(\mathrm{mg} / \mathrm{kg})\end{array}$} & \multicolumn{2}{|c|}{ Monthly seizure frequency } \\
\hline & & & & & & & Placebo & $\begin{array}{l}\text { Last } 2 \text { months } \\
\text { on } V G B\end{array}$ \\
\hline 1 & $\mathbf{F}$ & 32 & $\mathbf{C P}$ & CBZ 1800 PB 150 & 44 & 41 & $9 \cdot 5$ & 2 \\
\hline 2 & $\mathrm{~F}$ & 31 & $\mathrm{CP}$ & CBZ 1000 PB 100 & 47 & 50 & 7 & $1 \cdot 5$ \\
\hline 3 & $\mathbf{M}$ & 25 & $\mathrm{CP}+\mathrm{SG}$ & CBZ 1600 PB 150 & 45 & 47 & 95 & 39 \\
\hline 4 & $\mathbf{M}$ & 41 & $\mathrm{CP}$ & CBZ 1200 & 35 & 25 & 6 & 0 \\
\hline 5 & $\mathbf{M}$ & 41 & $\mathbf{C P}$ & PHT 450 VPA 600 & 29 & 46 & 4 & 0.5 \\
\hline 6 & $\mathbf{F}$ & 29 & $\mathbf{C P}$ & CBZ 1200 PB 150 & 26 & 52 & 10 & $3 \cdot 5$ \\
\hline 7 & $\mathbf{F}$ & 31 & $\mathrm{CP}$ & CBZ 1200 & 34 & 38 & $12 \cdot 5$ & $4 \cdot 5$ \\
\hline $8^{*}$ & $\mathbf{M}$ & 28 & $\mathbf{C P}$ & CBZ 1200 & 16 & 60 & 11 & 6 \\
\hline $9^{*}$ & $\mathbf{M}$ & 41 & CP & CBZ 1000 PB 150 & 4 & 31 & 7 & 4.5 \\
\hline $10^{*}$ & $\mathbf{M}$ & 18 & $\mathrm{SP}+\mathrm{SG}$ & CBZ 1200 PB 125 & 8 & 29 & $24 \cdot 5$ & $6 \cdot 5$ \\
\hline $11^{*}$ & $\mathbf{M}$ & 19 & AT & CBZ 600 VPA 1700 & 6 & 39 & $35 \cdot 5$ & 32 \\
\hline $12^{*}$ & $\mathbf{M}$ & 37 & ABS & PB 175 ETSM 1250 & 15 & 31 & 6 & 2 \\
\hline $13^{*}$ & $\mathbf{F}$ & 26 & $\mathrm{CP}+\mathrm{SG}$ & CBZ 800 PB 200 & 1 & 59 & 20 & early drop-out \\
\hline 14 & $\mathbf{M}$ & 26 & $\mathrm{CP}$ & CBZ 1200 PB 150 & 31 & 39 & $4 \cdot 5$ & 0 \\
\hline 15 & $\mathbf{F}$ & 42 & CP & CBZ 800 CLBZ 20 & 19 & 38 & 8 & $2 \cdot 5$ \\
\hline 16 & $\mathbf{F}$ & 52 & $\mathrm{CP}+\mathrm{SG}$ & PHT 350 PB 200 & 24 & 47 & 3 & 0.5 \\
\hline 17 & $\mathbf{M}$ & 24 & $\mathrm{CP}+\mathrm{SG}$ & CBZ 1800 PB 200 & 24 & 48 & 30 & 10 \\
\hline $18^{*}$ & $\mathbf{M}$ & 20 & $\mathrm{CP}$ & CBZ 1200 & 18 & 58 & $5 \cdot 5$ & 3 \\
\hline 19 & $\mathbf{F}$ & 23 & $\mathrm{CP}$ & CBZ 1200 PB 150 & 25 & 40 & $6 \cdot 5$ & 0.5 \\
\hline 20 & $\mathbf{M}$ & 56 & SP & PHT 400 PB 125 & 22 & 30 & $5 \cdot 5$ & 1 \\
\hline 21 & $\mathbf{F}$ & 25 & $\mathrm{TC}$ & VPA 1750 PB 100 & 22 & 64 & $11 \cdot 5$ & $4 \cdot 5$ \\
\hline 22 & $\mathbf{M}$ & 59 & $\mathrm{CP}$ & CBZ 1000 & 24 & 48 & 98 & $5 \cdot 5$ \\
\hline $23^{*}$ & $\mathbf{F}$ & 34 & $\mathrm{CP}$ & PHT 250 PB 150 & 14 & 69 & 68 & 74 \\
\hline 24 & $\mathbf{F}$ & 40 & $\mathrm{CP}$ & PHT 300 VPA 600 & 18 & 74 & 18 & 9 \\
\hline 25 & $\mathbf{F}$ & 22 & $\mathrm{CP}+\mathrm{SG}$ & CBZ 1000 PB 100 & 15 & 57 & $48 \cdot 5$ & 48 \\
\hline $\begin{array}{l}\text { Median } \\
\text { (range) }\end{array}$ & \multicolumn{4}{|c|}{$31(18-58)$} & $22(1-47)$ & $47(25-74)$ & $10(3-98)$ & $4 \cdot 5(0-74)$ \\
\hline
\end{tabular}

*drop-out $(\mathrm{ABS}=$ absence, $\mathrm{AT}=$ atonic, $\mathrm{CP}=$ complex partial, $\mathrm{SP}=$ simple partial, $\mathrm{SG}=$ secondary generalisation, $\mathrm{TC}=$ tonic clonic), drug treatment and response to long-term vigabatrin (VGB) in the patients included in the study. Drug abbreviations used: $\mathrm{CBZ}=$ carbamazepine, $\mathrm{CLBZ}=$ clobazam, ETSM = ethosuximide, PB = phenobarbitone, PHT $=$ phenytoin, VPA $=$ sodium valproate

patients went directly into the long-term phase of the study without discontinuation of the initial VGB treatment (in this group, overall duration of treatment includes this initial period).

Each patient was given the option to continue VGB indefinitely if the drug was felt to be beneficial. The dosage of associated anticonvulsants was maintained unchanged throughout. Seizure frequency was recorded prospectively by the patient on appropriate fit charts. Each patient was seen regularly in the clinic at 3 months intervals. Routine urinalysis, haematology, blood chemistry and serum anticonvulsant drug levels (TDx, Abbott) were obtained at each visit. Visual, brainstem auditory and somatosensory evoked potentials were recorded ${ }^{8}$ before starting VGB and at 6month intervals thereafter.

Statistical analysis Median monthly seizure frequencies were calculated for consecutive 2-month periods throughout the duration of follow-up and compared with the corresponding values recorded in the same patients during the initial placebo-period. Since these data showed a non-normal distribution, comparisons were made by using the paired Wilcoxon's rank test. For each subject, the percent change in mean seizure frequency between the placebo-period and the follow-up phase was also tested by paired Student's $t$ test.

Serum anticonvulsant levels before and after VGB in each patient and for all patients as a group were compared by paired $t$ test. Comparisons of evoked potentials parameters among treatment periods were made by analysis of variance and by paired $t$ tests with Bonferroni's correction.

\section{Results}

Drop outs and duration of follow-up Eight patients dropped out of the study, one after 1 month because of ataxia (which rapidly subsided after discontinuation of VGB), one after 16 months because of psychotic symptoms, three after 6,14 and 18 months because of seizure breakthrough and three after 4,8 and 15 months for reasons unrelated to treatment. The duration of treatment in the 17 patients who continue on VGB ranges from 14 to 47 months (median 22 months).

Efficacy data During the first two months of longterm VGB treatment, the median seizure frequency dropped to less than one half that observed during the placebo period and remained significantly reduced throughout the full duration of follow-up (fig 1). Although the apparent improvement in seizure control after 2 years of follow-up reflects partly a bias effect related to a progressively decreasing number of patients, the persistence of seizure reduction during VGB therapy could be clearly confirmed by plotting separately the data from the 21 patients who received VGB for at least 14 consecutive months (fig 1 , inset). The persistence of the improved clinical control was also clearly apparent by examining the percent change 


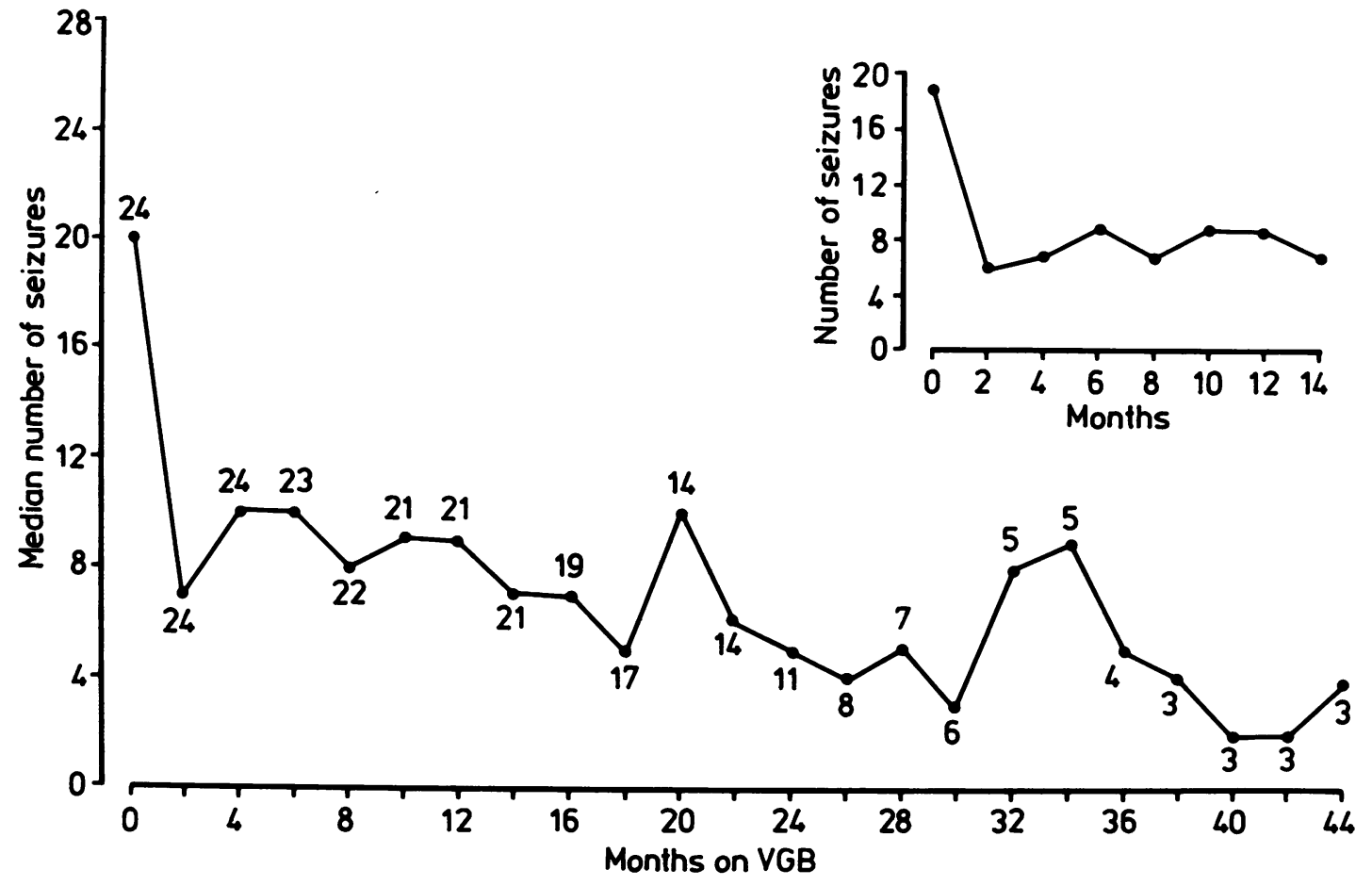

Fig 1 Median seizure frequency (number of fits/2 months) during the initial placebo period (time 0 ) and at different intervals during long-term vigabatrin (VGB) therapy. Numbers indicate the number of patients in each period (data from the patient who dropped out after 1 month were excluded). Inset: Same data for the 21 patients who completed 14-month treatment.

in seizure frequency (compared to placebo) for each patient (fig 2). Again, the reduction in fit frequency was statistically significant at each assessable time point. The trend towards a better seizure control with increasing duration of treatment is partly due to the fact that the best responders tended to be treated for longer: in at least five patients, however, there was a clear-cut further improvement in fit frequency after several months of treatment, partly as a result of increased VGB dosage.

Individual seizure frequencies on placebo and during the last two months on VGB (including patients who dropped out) are reported in table 1 . The monthly median seizure frequency was 10 on placebo and 4.5 on VGB $(p<0.01)$. In the 17 patients who remain in the trial, the monthly median seizure frequency during the last 60 days of follow up was 2.5 (range 0-48) compared with 9.5 (range 3-98) during placebo. Among these patients, two have now been completely seizure-free for the last 20 and 30 months respectively.

When responses in relation to seizure type were considered, the effect of VGB on complex partial seizures overlapped closely with that reported above for all seizures grouped together. Individual responses in patients with different seizures types are given in table 1; the number of these patients, however, was too small to allow a meaningful evaluation by specific seizure type.

Adverse effects Side effects reported during VGB treatment were usually mild and are listed in table 2 . The most frequently observed of these effects was weight gain, which ranged from 3 to $11 \mathrm{~kg}$ ( 5 to $16 \%$ of the initial body weight). With the exception of weight gain, which occurred usually after 3-6 months of VGB therapy and tended to plateau within a few months, side effects were detected in a similar proportion of patients throughout the study. Only two patients discontinued VGB because of possibly drug-related adverse effects (ataxia and psychotic symptoms respectively) and only one patient required a reduction of VGB dosage because of suspected toxicity. Positive side effects (improved mood) were reported by four patients.

Evoked potentials Somatosensory evoked potential parameters remained virtually unchanged during VGB treatment. There was a trend for some brainstem auditory potential latencies to decrease slightly during long-term VGB. P100 latencies of visual evoked potentials were abnormally prolonged in many 


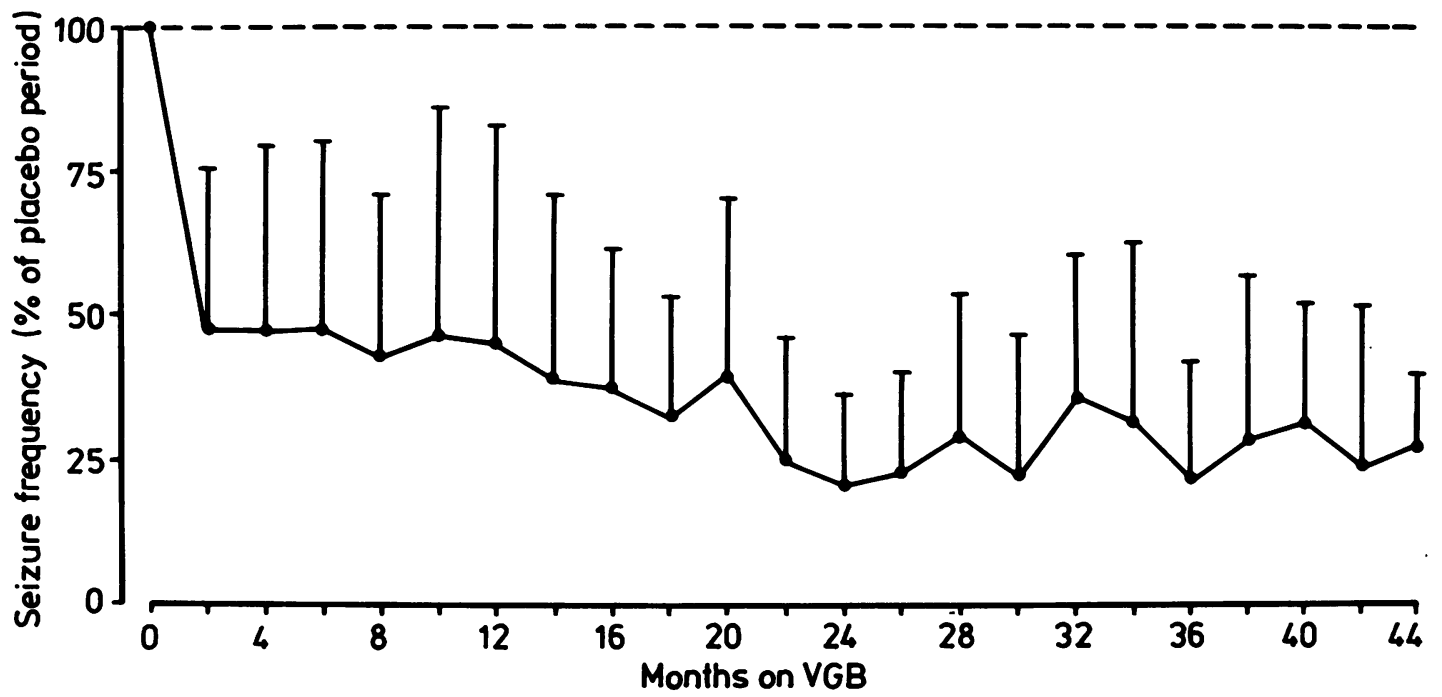

Fig 2 Percent change in seizure frequency (vs placebo) at different intervals during long-term vigabatrin (VGB) therapy. Symbols represent means, s.d. Numbers of patients at each time point as in fig 1 .

patients before $\mathrm{VGB}^{8}$ and showed a trend towards normalisation during treatment.

Serum anticonvulsant drug levels Before starting VGB, serum drug levels $(\mu \mathrm{g} / \mathrm{ml}$, means, s.d.) were $7 \cdot 8$, $2 \cdot 7$ for carbamazepine $(n=18), 28 \cdot 7,7 \cdot 8$ for phenobarbital $(n=16), 13 \cdot 8,4 \cdot 2$ for phenytoin $(n=5)$ and 48,16 for valproic acid $(n=3)$. When all patients were considered together, mean serum carbamazepine, phenobarbital and valproic acid levels were not significantly affected by VGB, whereas serum phenytoin levels decreased significantly to $9 \cdot 4,4 \cdot 2 \mu \mathrm{g} / \mathrm{ml}(\mathrm{p}<$ $0.05)$.

Table 2 Side effects recorded during VGB treatment

\begin{tabular}{lc}
\hline Weight gain & 10 \\
Sedation & 4 \\
Headache & 4 \\
Epigastric pain, nausea & 4 \\
Weakness & 3 \\
Anxiety, irritability & 3 \\
Vertigo & 3 \\
Dysarthria & 2 \\
Confusion & 2 \\
Ataxia & $1^{*}$ \\
Psychotic symptoms & $1^{*}$ \\
Depression & 1 \\
Insomnia & 1 \\
Anorexia & 1 \\
Diarrhea & 1 \\
Itching & 1 \\
Fever & 1 \\
Increased nail fragility & 1 \\
Improved mood & 4 \\
\hline
\end{tabular}

Numbers indicate number of patients reporting a given effect. *denotes effect resulting in drop-out.

\section{Discussion}

For ethical reasons, only patients who had shown a clear-cut favorable response to VGB were selected for long-term treatment. Therefore, the study was not intended to assess the proportion of patients showing a satisfactory response but, rather, to obtain information on the risk/benefit ratio during maintenance therapy. At each study visit, the decision on whether to continue VGB was thoroughly discussed with each patient and treatment was maintained only when clinical data indicated significant therapeutic benefit. For this reason, the fact that 17 out of 25 patients are still on VGB after a period of up to 4 years is by itself an indication that the drug retained its efficacy. This conclusion is reinforced by the observation that only five patients dropped out because of possible adverse effects or seizure breakthrough.

The persistent efficacy of VGB could be documented objectively by seizure frequency recording throughout follow-up. Compared with the placebo period, the mean percent reduction in seizure frequency after initial exposure to VGB (blind phase preceding follow-up) in these patients was 56, SD $29 \%$. This compares with a mean reduction of $60, \mathrm{SD}$ $29 \%$, observed during the last 2 months of long-term therapy in the same subjects, including those who dropped-out. Although adjustments in VGB dosage were allowed during long-term therapy, the dosage of the drug during the last 2 months of follow-up (3.0, SD $0 \cdot 8 \mathrm{~g} /$ day) was not markedly different from that used during the initial blind phase of the study $(2 \cdot 6, \mathrm{SD}$ 
$0.6 \mathrm{~g} /$ day). Moreover, changes in dosage were generally made during the first 14 months of treatment and overall seizure control showed no trend to deteriorate after this period (in fact, upwards dosage adjustments generally resulted in further improvement in fit frequency).

It might be argued that the sustained seizure control observed during long-term VGB could be partly related to a "regression towards the mean" phenomenon, but we feel that this is unlikely for at least two reasons. First, in almost half of the patients the initial response to VGB was assessed by using a double-blind design which included a randomised placebo-active drug sequence. ${ }^{4}$ Second, the depression of seizure frequency during VGB therapy was consistent throughout the long duration of follow-up (figs 1 and 2), without the relapses expected if the initial response was due to spontaneous variation in the severity of the seizure disorder.

Side effects were usually mild, the most frequent of them being weight gain. Possibly drug-related effects leading to VGB withdrawal were seen in only two cases. In the patient who developed ataxia after 1 month on VGB, the causative role of the drug is questionable because this subject had tolerated previously VGB for 7 weeks without untowards effects. The second drop-out involved a 42 year man who, after 14 months of good seizure control, developed psychotic symptoms which did not clear after VGB withdrawal and required neuroleptic treatment. Appearance or worsening of psychotic symptoms have been occasionally reported in previous trials of $\mathrm{VGB}^{79}$ and may thus represent a real side effect of the drug in susceptible patients.

- Toxicological studies have detected vacuolisation in the white matter of the brain of laboratory animals receiving relatively large doses of VGB.' In the present study, we observed no clinical symptoms suggestive of serious neurological damage. In addition, we found no deterioration in brainstem auditory, somatosensory and visual evoked potential parameters, an observation which is especially relevant because clear-cut evoked potential alterations parallel the time course of VGB-induced neurotoxicity in experimental animals. ${ }^{10}$ Serum levels of associated anticonvulsants were not affected by VGB, except for phenytoin levels which decreased in some patients, in agreement with previous findings. ${ }^{267}$

In conclusion, our results provide clear evidence that VGB retains anticonvulsant efficacy and good tolerability during long-term treatment. These data supplement those recently reported by Browne et $\mathrm{al}^{7}$ in a similar population and underline the potential usefulness of this new drug for the management of epilepsy.

\section{References}

1 Hammond EJ, Wilder BJ. Gamma-vinyl-GABA: a new antiepileptic drug. Clin Neuropharmacol 1985;8:1-12.

2 Rimmer EM, Richens A. Double-blind study of gammavinyl-GABA in patients with refractory epilepsy. Lancet 1984;1:189-90.

3 Gram L, Klosterkov P, Dam M. Gamma-vinyl-GABA: a double-blind placebo-controlled trial in partial epilepsy. Ann Neurol 1985;17:262-6.

4 Loiseau P, Hardenberg JP, Pestre M, Guyot M, Schechter PJ, Tell GP. Double-blind, placebo-controlled study of vigabatrin (gamma-vinyl-GABA) in drugresistant epilepsy. Epilepsia 1986;27:115-20.

5 Tartara A, Manni R, Galimberti CA, Hardenberg J, Orwin J, Perucca E. Vigabatrin in the treatment of epilepsy: A double-blind, placebo-controlled study. Epilepsia 1986;27:717-23.

6 Tassinari CA, Michelucci R, Ambrosetto G, Salvi F. Double-blind study of vigabatrin in the treatment of drug resistant epilepsy. Arch Neurol 1987;44:907-10.

7 Browne TR, Mattson RH, Penry JK, et al. Vigabatrin for refractory complex partial seizures: Multicenter singleblind study with long-term follow-up. Neurology 1987;37:184-9.

8 Cosi V, Callieco R, Galimberti CA, Manni R, Tartara A, Lanzi G, Balottin U, Perucca E. Effect of vigabatrin (gamma-vinyl-GABA) on visual, brainstem auditory and somatosensory evoked potentials in epileptic patients. Eur Neurol 1987;28:42-46.

9 Korsgaard S, Casey D, Gerlach J. Effect of gamma-vinylGABA in tardive dyskinesia. Psychiatry Res 1983; 8:261-269.

10 Arezzo JC. Effect of vigabatrin on evoked potentials in dogs. Br J Clin Pharmacol 1989 (in press). 